\title{
A GESTÃO DA QUALIDADE DOS ALIMENTOS NOS SERVIÇOS DE GASTRONOMIA NA CIDADE DE SÃO PAULO DURANTE A PANDEMIA DE COVID 19
}

\section{FOOD QUALITY MANAGEMENT IN GASTRONOMY SERVICES IN THE CITY OF SÃO PAULO DURING THE COVID 19 PANDEMIC}

\author{
Renata Pereira Takamatsu ${ }^{1}$ \\ Pós-graduação (Saúde Pública com ênfase em Saúde da Família e Psicopedagogia) \\ Universidade Anhembi Morumbi, São Paulo, SP, Brasil. \\ https://orcid.org/0000-0002-3272-2273 \\ rpt.nutricionista@gmail.com
}

${ }^{1}$ Administração do projeto, curadoria, análise e interpretação de dados, metodologia, escrita, edição e revisão

\section{RESUMO}

Devido ao risco da pandemia COVID 19, foram adotadas estratégias para reduzir as taxas de contágio das populações e tentar evitar crise no sistema de saúde. A categoria de bares e restaurantes busca adequar-se a essas estratégias, mantendo a qualidade e higiene de seus serviços para colaboradores e cliente. Neste trabalho 0 objetivo foi realizar 0 levantamento e análise sobre as ações direcionadas à prevenção da transmissão da COVID 19 em serviços de alimentação. A metodologia adoada foi a revisão bibliográfica por meio de dados disponíveis em bases de dados científicos como: Scielo, PubMed e Órgãos do Governo, nos últimos 43 anos. O período de busca se deu entre dezembro de 2020 e fevereiro de 2021 e resultou em 28 artigos. O Governo de São Paulo determinou fases de acordo com o avanço da pandemia e cada uma delas apresenta restrições para bares e restaurantes, além disso, outros autores desenvolveram orientações para que eles pudessem seguir o padrão das boas práticas de higiene nos serviços de alimentação. Concluiu-se que há necessidade de manter a qualidade higiênico sanitária em serviços de gastronomia de São Paulo, também é importante que os clientes sejam ouvidos e que funcionários tenham treinamentos para evitar a contaminação da COVID 19 e manter as pessoas sadias.

Palavras-chave:

Administração.

\begin{abstract}
Due to the risk of the COVID 19 pandemic, strategies were adopted to reduce the contagion rates of populations and try to avoid a crisis in the health system. The category of bars and restaurants seeks to adapt to these strategies, maintaining the quality and hygiene of its services for employees and customers. In this work, the objective was to carry out a survey and analysis of actions aimed at preventing the transmission of COVID 19 in food services. The adopted methodology was a bibliographic review using data available in scientific databases such as: Scielo, PubMed and Government Agencies, in the last 43 years. The search period took place between December 2020 and February 2021 and resulted in 28 articles. The Government of São Paulo determined phases according to the progress of the pandemic and each one of them presents restrictions for bars and restaurants, in
\end{abstract}


addition, other authors developed guidelines so that they could follow the standard of good hygiene practices in food services. It was concluded that there is a need to maintain the hygienic and sanitary quality in gastronomy services in São Paulo, it is also important that customers are heard and that employees have training to avoid contamination of COVID 19 and keep people healthy.

Keywords: Administration. Food. Corona Virus

\section{INTRODUÇÃO}

No início do ano de 2020, a Organização Mundial de Saúde (OMS) declarou emergência de Saúde Pública à pandemia causada pela COVID-19 (CRODAL et. al., 2020; CHEN et. al. 2020) criando ações estratégicas e coordenadas por parte dos governos, tendo em mitigação dos impactos da contaminação (CRODAL et al., 2020). Foram adotadas medidas como fechamento de alguns serviços (incluindo 0 setor da gastronomia), prática do distanciamento social, que reduziu as taxas de transmissão e de mortalidade nos locais em que foram utilizadas.

No atual momento em que as pessoas vivem, a cadeia de alimentos é essencial e, por isso não pode parar. $O$ SarS-Cov2, responsável pela atual pandemia, é um vírus transmitido de pessoa para pessoa por contato próximo (CHU et al. 2020) e até o momento, não há evidências que a Covid 19 possa ser transmitida através de alimentos ou embalagens alimentícias, pois para que ocorra a multiplicação do vírus, é necessário um hospedeiro animal ou humano (OMS/FAO, 2020). Alimentos devem ser servidos aos clientes com a garantia da segurança dos alimentos e que devem assegurar a saúde dos colaboradores.

\section{Gestão e qualidade:}

A qualidade de serviço pode ser determinada pelas expectativas e percepções dos clientes sobre o serviço oferecido (EL-SAID e FATHY, 2015). A satisfação do cliente é um dos principais determinantes da lucratividade a longo prazo de uma empresa e da lealdade do cliente (SPYRIDOU, 2017). Por isso é importante que a retomada das atividades gastronômicas nesse período pandêmico seja realizada com segurança e qualidade para clientes e funcionários.

A Figura 1 mostra como deve ser feita a avaliação do serviço de gastronomia pelo cliente, com expectativas e percepções de qualidade ideal, satisfatória ou inaceitável de cada um.

Figura 1 - Avaliação da qualidade do serviço de gastronomia pelos clientes

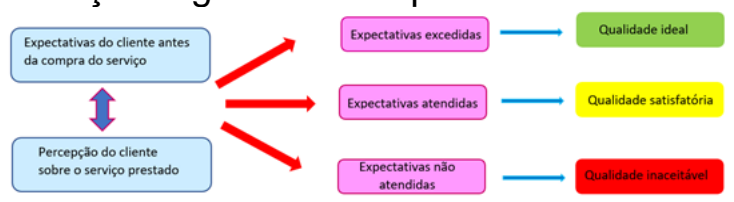

(TAKAMATSU, 2021)

Há uma tendência natural às percepções sobre os serviços de alimentação serem desfavoráveis. Vários fatores são responsáveis por 
isso, como cardápio limitado, baixa qualidade da comida e do serviço, facilidade e a variedade em geral (KWUN, 2011). Aspectos higiênicos de manipuladores de alimentos e ambiente alimentar tiveram impacto significativo e alto na satisfação com a qualidade do serviço alimentar (TAKALKAR, KUMAVAT, 2011; DONKOH et al., 2012; NADZIRAH et al., 2013). Um grande risco de contaminação de alimentos está associado aos manipuladores de alimentos (TAKALKAR, KUMAVAT, 2011).

\section{METODOLOGIA}

A pesquisa foi realizada por meio de revisão bibliográfica desenvolvida em publicações cientificas, Decretos Estaduais e livros. Para a realização da pesquisa foi efetuado um levantamento de publicações relacionadas com o tema em documentos de língua portuguesa, inglesa e espanhola, indexados em bases de dados e publicados nos últimos 43 anos. Para realização da pesquisa foram consultadas as bases de dados: Scielo (Scientific Electronic Library Online), PubMed (Nation Library of Medicine), Biblioteca Virtual em Saúde, Órgãos do Governo e da Saúde etc. Os descritores utilizados foram: Pandemia, Covid-19, Corona vírus, administração, bares e restaurantes, qualidade, gestão. O período de busca foi de dezembro de 2020 até fevereiro de 2021 e resultou em 28 artigos. O critério de inclusão, para o presente estudo, foi o acesso aos artigos, Leis, Decretos e Resoluções na íntegra, nos idiomas português, inglês e espanhol, com uma análise criteriosa da qualidade dos mesmos, que abordassem temas como gestão, qualidade dos serviços, Covid-19, qualidade dos alimentos, cuidados com a pandemia e os estudos que não abordavam esses temas foram excluídos.

\section{RESULTADOS E DISCUSSÃO}

\section{Segurança dos Alimentos:}

A segurança dos alimentos é um desafio à saúde pública, visa oferecer alimentos isentos de contaminantes aos cidadãos e a manter a integridade da saúde do consumidor (SIEBENEICHLER et al., 2007). Dentre os principais fatores relacionados a Doenças de Origem Alimentar (DTAs), destacam-se: más condições de higiene na manipulação, armazenamento e conservação dos alimentos; uso incorreto do binômio tempotemperatura; falta de adequação e conservação da estrutura física dos estabelecimentos; etc (STEFANINI, ALVES, MARQUES, 2018). Diante da pandemia, a discussão sobre segurança dos alimentos e suas interfaces, tendo em vista o que já ocorreu nos países europeus, é de extrema urgência. 
As normativas publicadas catalisaram o fechamento de muitos estabelecimentos de refeições coletivas e a migração de outros tantos para os serviços de take out/away e delivery. Essa adaptação dos serviços teve como foco principal a sobrevivência desse setor da economia no momento de crise. As empresas buscam manter os custos fixos, como salários de funcionários e aluguéis, bem como otimizar os custos variáveis. Para melhorar esse cenário, a Abrasel (2020a) fechou uma parceria com uma empresa do ramo de entrega de refeições, cujos pedidos são realizados via internet. Além disso, também publicou orientações aos empresários em relação aos cuidados de higiene no delivery (ABRASEL, 2020b). Se por um lado a entrega em domicílio é uma solução viável para minimizar a crise do setor, por outro, sabe-se que essa tecnologia não está acessível a toda população. A precarização do trabalho, que inclui as atividades realizadas por meio das plataformas virtuais, aumenta a vulnerabilidade dos entregadores e os coloca na linha de frente de exposição ao SARS-CoV-2.

Com objetivo de evitar a insegurança dos alimentos, e problemas decorrentes, se faz necessário a utilização de fluxo que contempla as etapas de controle higiênico sanitário. A legislação estadual, através de Código sanitário de São Paulo, preconiza promoção, preservação e recuperação da saúde pública e, consequentemente a melhoria contínua das Normas Técnicas Especiais de alimentos e bebidas, envolvendo:

a) Segurança dos alimentos destinados ao consumo humano;

b) Higiene Ambiental de produção e armazenagem nas áreas de cultivo $e$ produção de matéria-prima e de alimento in natura;

c) Condições Sanitárias de transportes;

d) Construção e seus requisitos para estabelecimentos com práticas operacionais;

e) Requisitos para equipamentos e utensílios;

f) Higiene nas operações e produção;

g) Programas de Controle Sanitário e de Laboratório;

h) Especificação do produto acabado através da rotulagem.

(SÃO PAULO, 1978a; SÃO PAULO, 1978b)

Os aspectos legais são referências essenciais para o bom desempenho e aplicabilidade diária da rotina dos serviços de gastronomia e consistem em forma positiva, na segurança dos alimentos é fundamental para que haja alcance da gestão da qualidade. Ocorre que a maioria dos estabelecimentos de alimentação na cidade de São Paulo podem ser considerados microempresas ou empresas de pequeno porte e possuem maior dificuldade em atender a todas as normas e legislações vigentes na cidade de São Paulo a respeito dos padrões de higiene 
exigidos pela Secretaria de Saúde, além da legislação estadual e federal, como a RDC 218/04- Anvisa.

É importante que haja um planejamento de ações para o enfrentamento da crise gerada pela Covid19 afim de não afetar de forma negativa o setor gastronômico e poder continuar garantindo a qualidade dos serviços prestados, segurança dos alimentos, saúde de funcionários e clientes, respeitando todas as novas regras para impedir a disseminação do vírus entre a população atendida no chamado "novo normal". A gestão da vigilância sanitária para a maior segurança do investidor gastronômico, evitando-se desinformação, multas, fechamentos, comprometimentos da saúde de consumidores ou funcionários passa a ser fundamental e a constante atualização, assim como mudanças no plano de ação de acordo com a mudança de situação é essencial para o sucesso.

Treinamentos online, simulações são considerados opções importantes no desenvolvimento empresarial e profissional e durante essa fase de pandemia passam a fazer parte da rotina de muitas empresas e seus profissionais. Com o aumento do trabalho remoto, as reuniões passam a ser reconfiguradas. As simulações adquiriram outros formatos, com máscaras, luvas, roupas apropriadas, álcool gel $70 \%$ e distanciamento social, Chu et. al (2020) acredita que a proteção para os olhos está relacionada com menos infecções do
Corona Vírus. O ambiente passa a ser rigorosamente higienizado em intervalo de tempo menor do que anteriormente, assim como móveis e utensílios. A higiene e manipulação de alimentos, vigilância sanitária, saúde e segurança no trabalho são tópicos permanentes do projeto de educação continuada da entidade, contribuindo consideravelmente no treinamento e desenvolvimento empresarial e profissional. A higiene alimentar faz parte da evolução da produtividade e qualidade total no atendimento ao cliente (STEFANINI, ALVES, MARQUES, 2018), condição prioritária para a retomada dos negócios em 2021 com alto grau de sustentabilidade empresarial. É importante frisar a estrutura e organização necessárias para a melhoria da gestão da qualidade de higiene alimentar para restaurantes e similares com foco nas Boas Práticas de Fabricação (BPF). Nesse sentido, realça-se rigorosamente a garantia e busca da qualidade, os direitos básicos do consumidor, além da criação de um ambiente de trabalho mais produtivo e satisfatório para os colaboradores envolvidos no processo.

Assim, são detalhados inúmeros aspectos para a melhor estruturação e organização da higiene alimentar, merecendo-se destaque: o ambiente, os equipamentos e utensílios, os manipuladores, alimentos, instalações sanitárias e o controle de pragas (WILDER- 
SMITH, FREEDMAN, 2020). É importante analisar, a adoção sistêmica das Boas Práticas como um dos mais relevantes instrumentos para o melhor alcance da qualidade, da segurança dos alimentos e excelência global do produto.

Figura 2 - Estratégias para 0 enfrentamento da COVID-19 no âmbito dos programas de segurança dos alimentos e bebidas

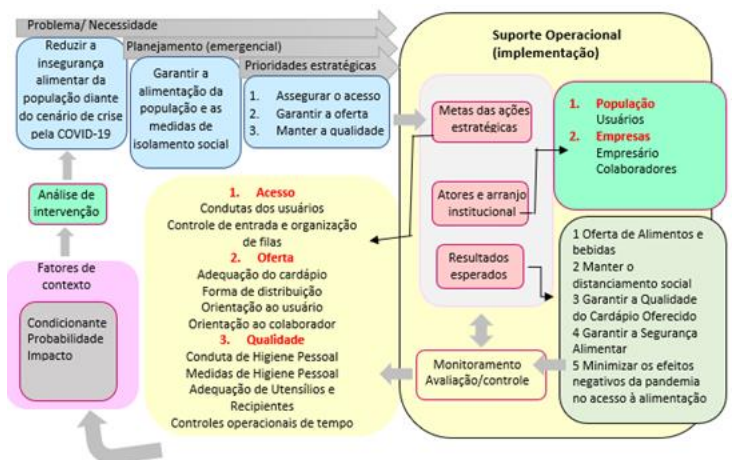

(TAKAMATSU, 2021)

Além disso, estabelecer um checklist e uma rotina de higienização para a melhoria do gerenciamento da atividade produtiva. Nesse contexto, os manipuladores de alimentos são os protagonistas dessa nova realidade, com treinamento e avaliação constantes das equipes, controle periódico de saúde das mesmas e a melhoria contínua do grau de instrução dos funcionários para uma gestão eficaz (ABRANCHES, OLIVEIRA, SANTOS, 2020).

Em meio a pandemia, os donos de estabelecimentos como bares e restaurantes devem estar atentos as fases determinadas pelo governo: vermelha, laranja, amarela e verde, bem como os decretos, que informam como bares, restaurantes e similares devem funcionar em todas as fases propostas pelo Governo do Estado de São Paulo, a quadro 1 mostra a primeira determinação do Governo, e quadro 2, a determinação vigente.

Quadro 1 - Atendimento fase 1 e 2 vermelha e laranja, 2020

\begin{tabular}{|l|c|c|}
\hline $\begin{array}{l}\text { Atendimento } \\
\text { presencial }\end{array}$ & Fase 1 & Fase 2 \\
\hline $\begin{array}{c}\text { Consumo local } \\
\text { (Bares, restaurantes } \\
\text { e similares) }\end{array}$ & $\mathrm{X}$ & $\mathrm{X}$ \\
\hline
\end{tabular}

Quadro 2 - Atendimento fase 3 amarela, 2020

\begin{tabular}{|l|l|}
\hline $\begin{array}{l}\text { Atendimento } \\
\text { presencial }\end{array}$ & Fase 3 \\
\hline & $\begin{array}{l}\text {-Somente ao ar } \\
\text { livre }\end{array}$ \\
& $\begin{array}{l}\text { C Capacidade } 40 \% \\
\text { limitada }\end{array}$ \\
$\begin{array}{c}\text { Consumo local } \\
\text { (Bares, } \\
\text { restaurantes e } \\
\text { similares) }\end{array}$ & $\begin{array}{l}\text { - Horário reduzido } \\
\text { (6 horas seguidas) } \\
\text { - Adoção dos } \\
\text { protocolos padrões } \\
\text { e setoriais } \\
\text { específicos. }\end{array}$ \\
\hline
\end{tabular}

(SÃO PAULO, 2020)

O Centro de Vigilância Epidemiológica e o Centro de Contingências do Coronavírus, que fazem parte da Secretaria de Saúde, são responsáveis por realizarem a monitoração do progresso da pandemia do Corona Vírus no Estado, restringindo ou flexibilizando as atividades e serviços não essenciais. 
No dia 23 de março de 2020 foi criado o Decreto no 64.881 , que decretou a quarentena em todo o Estado de São Paulo em razão da pandemia de COVID 19, levando em conta o Decreto Federal 10.282, de 20 de março de 2020, que regulamenta a Lei no 13.979, de 6 de fevereiro de 2020, para definir os serviços públicos e as atividades essenciais, dentre eles a produção, distribuição, comercialização e entrega, de forma presencial ou eletrônica de alimentos e bebidas [...]. Além de seguirem as determinações dos decretos, devem atentar nas medidas especiais que protegem idosos, gestantes e pessoas com doenças crônicas ou imunodeprimidas, de acordo com as recomendações da Secretaria de Estado da Saúde e do Ministério da Saúde, além de impedir aglomerações.

Quadro 3 - Atendimento fase 4 verde, 2020

\begin{tabular}{|l|l|}
\hline $\begin{array}{l}\text { Atendimento } \\
\text { presencial }\end{array}$ & Fase 4 \\
\hline Consumo local \\
$\begin{array}{c}\text { (Bares, } \\
\text { restaurantes e } \\
\text { similares) }\end{array}$ & $\begin{array}{l}\text { - Capacidade } 60 \% \\
\text { limitada }\end{array}$ \\
& $\begin{array}{l}\text { Adoção dos } \\
\text { protocolos } \\
\text { padrões e } \\
\text { setoriais } \\
\text { específicos. }\end{array}$ \\
\hline
\end{tabular}

(SÃO PAULO, 2020)
Quadro 4 - Atendimento fase 1 e 2, vermelha e laranja, 2021

\begin{tabular}{|c|c|c|}
\hline $\begin{array}{l}\text { Atendimento } \\
\text { presencial }\end{array}$ & $\begin{array}{c}\text { Fase } \\
1\end{array}$ & Fase 2 \\
\hline $\begin{array}{l}\text { Consumo } \\
\text { local } \\
\text { (restaurantes } \\
\text { e similares }\end{array}$ & $X$ & $\begin{array}{l}\text { - Capacidade } 40 \% \\
\text { limitada } \\
\text { - Horário reduzido } \\
\text { (8 horas): Após as } \\
6 \text { 6h antes das } 20 \mathrm{~h} \\
\text { - Consumo local e } \\
\text { atendimento } \\
\text { exclusivo para } \\
\text { clientes sentados } \\
\text { - Venda de } \\
\text { bebidas alcóolicas } \\
\text { até as } 20 \mathrm{~h} \\
\text { - Adoção dos } \\
\text { protocolos gerais } \\
\text { e setoriais } \\
\text { específicos }\end{array}$ \\
\hline $\begin{array}{c}\text { Consumo } \\
\text { local (Bares) }\end{array}$ & X & $\mathrm{x}$ \\
\hline
\end{tabular}

Quadro 5 - Atendimento fase 3, amarela, 2021

\begin{tabular}{|c|c|}
\hline $\begin{array}{l}\text { Atendimento } \\
\text { presencial }\end{array}$ & Fase 3 \\
\hline $\begin{array}{l}\text { Consumo local } \\
\text { (restaurantes e } \\
\text { similares) }\end{array}$ & $\begin{array}{l}\text { - Capacidade } 40 \% \\
\text { limitada } \\
\text { - Horário reduzido } \\
\text { (10 horas): Após as } \\
\text { 6h e antes das } 22 \mathrm{~h} \\
\text { - Consumo e } \\
\text { atendimento apenas } \\
\text { para clientes } \\
\text { sentados } \\
\text { - Venda de bebidas } \\
\text { alcóolicas até as } \\
\text { 20h } \\
\text { - Adoção dos } \\
\text { protocolos padrões } \\
\text { e setoriais } \\
\text { específicos. }\end{array}$ \\
\hline
\end{tabular}




\begin{tabular}{|c|c|}
\hline $\begin{array}{l}\text { Consumo local } \\
\text { (bares) }\end{array}$ & $\begin{array}{l}\text { - Capacidade } 40 \% \\
\text { limitada } \\
\text { - Horário reduzido } \\
\text { (10 horas): Após as } \\
6 \text { h e antes das } 20 \mathrm{~h} \\
\text { - Consumo e } \\
\text { atendimento apenas } \\
\text { para clientes } \\
\text { sentados } \\
\text { - Venda de bebidas } \\
\text { alcóolicas até as } \\
20 \text { h } \\
\text { - Adoção dos } \\
\text { protocolos geral e } \\
\text { setorial específicos }\end{array}$ \\
\hline
\end{tabular}

Quadro 6 - Atendimento fase 4, verde, 2021

\begin{tabular}{|c|c|}
\hline $\begin{array}{l}\text { Atendimento } \\
\text { presencial }\end{array}$ & Fase 4 \\
\hline $\begin{array}{l}\text { Consumo local } \\
\text { (restaurantes e } \\
\text { similares) }\end{array}$ & $\begin{array}{l}\text { - Capacidade } 60 \% \\
\text { limitada } \\
\text { - Horário reduzido } \\
\text { (12 horas): Após as } \\
6 \text { h e antes das } 22 \mathrm{~h} \\
\text { - Consumo e } \\
\text { atendimento apenas } \\
\text { para clientes } \\
\text { sentados } \\
\text { - Adoção de } \\
\text { protocolos geral e } \\
\text { setorial específicos }\end{array}$ \\
\hline $\begin{array}{l}\text { Consumo local } \\
\text { (bares) }\end{array}$ & $\begin{array}{l}\text { - Capacidade } 60 \% \\
\text { limitada } \\
\text { - Horário reduzido } \\
\text { (12 horas): Após as } \\
6 \text { h e antes das } 22 \mathrm{~h} \\
\text { - Consumo e } \\
\text { atendimento apenas } \\
\text { para clientes } \\
\text { sentados } \\
\text { - Adoção dos } \\
\text { protocolos geral e } \\
\text { setorial específicos }\end{array}$ \\
\hline
\end{tabular}

(SÃO PAULO, 2021)
A empresa Galunios e Qualibest (2020a) realizou uma pesquisa sobre a visão dos Operadores de FoodService sobre alimentação na pandemia, realizada entre os meses de novembro e dezembro de 2020, na qual mais da metade dos entrevistados reduziram suas vendas em mais de $26 \%$, fizeram pedido de empréstimo para instituições financeiras, demitiram parte da equipe (em torno de $37 \%$ dos trabalhadores foram mandados embora), a maioria dos responsáveis das empresas de alimentação acreditam que irão manter seus negócios após a pandemia. Eles também realizaram uma pesquisa para saber o comportamento do consumidor de alimentação fora do lar, no mês de julho de 2020, de fato, o consumidor está preocupado com a pandemia, pois receiam pela sua saúde, por isso que, muitos dos entrevistados colocam a higiene e limpeza como primeiro lugar de critério para a escolha dos restaurantes, não frequentam bares $e$ restaurantes durante a pandemia devido ao medo de pegar corona vírus e têm preferência por espaços abertos, que possuem messas nas calçadas, parques ou drive in e esperam que os responsáveis por restaurantes se preocupem com a qualidade do produto e que invistam em hospitalidade e segurança para o retorno dos mesmos (GALUNIOS, QUALIBEST, 2020b).

É importante ter atenção ao sistema de ar-condicionado dos estabelecimentos, 
os quais podem ser um mecanismo de transmissão do Corona Vírus, um dos primeiros casos relatados foi de um cliente de um restaurante que estava doente, mas ainda não tinha apresentado sintomas. Esse paciente estava sentado com sua família, próximos a saída de arcondicionado, sua mesa estava entre 2 mesas de famílias diferentes, no mesmo dia, o paciente caso índice apresentou seus primeiros sintomas e 12 dias depois, 9 pessoas entre das mesas A, B, C apresentaram os primeiros sintomas da COVID 19 (LU et al., 2020).

Figura 3 - Disposição das mesas

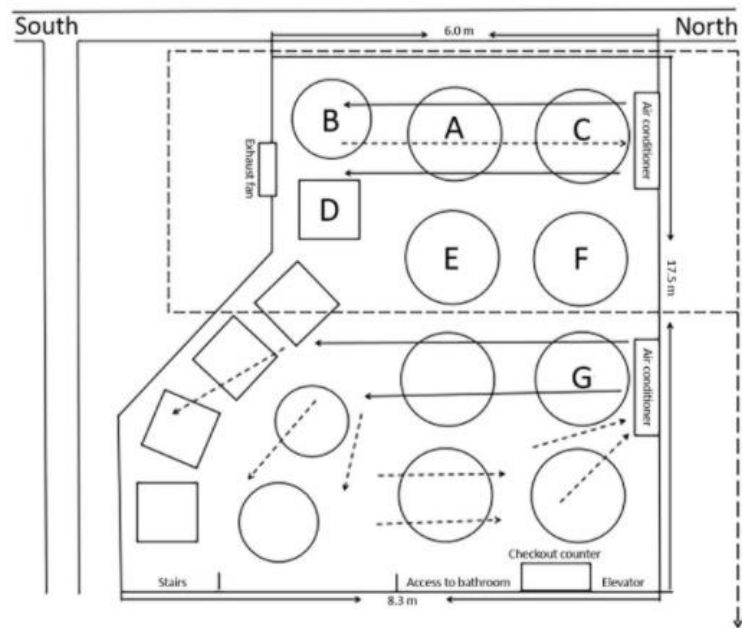

Representação da proximidade das mesas no restaurante próximas ao ar-condicionado. Distância entre as mesas $\mathrm{A}$ e $\mathrm{B}$ e $\mathrm{A}$ e $\mathrm{C}$ era de 1 metro (LU et al., 2020).

No caso do restaurante, a transmissão das gotículas do Sars Cov 2 foi conduzida através da ventilação do arcondicionado, o que indica que a causa da infecção foi o direcionamento do fluxo do ar. Os aerossóis costumam acompanhar o fluxo de ar e baixas concentrações de aerossóis, em grandes distâncias são incapazes de contaminar outros espaços, por isso, para reduzir a taxa de contaminação da COVID 19, bares e restaurantes devem melhorar a ventilação e aumentar o espaço entre as mesas (LU et al., 2020).

O atendimento presencial desses estabelecimentos deve ocorrer de forma responsável, sendo importante 0 cumprimento dos protocolos de segurança, para que não ocorra a disseminação do vírus, nem o ultrapasso do limite da capacidade hospitalar bem como a regressão das fases no Estado de São Paulo. Foram determinadas regras sobre a ocupação máxima dos estabelecimentos e restrição de horários. A Resolução RDC $n$ 2016 de 15 de setembro de 2004 continua em vigor, e tem o objetivo de garantir as boas práticas de higiene nos serviços de alimentação, da manipulação até a entrega ao cliente e além disso, a Universidade Estadual de Campinas - UNICAMP (2020) criou um guia para o convívio seguro, com orientações sanitárias para 0 enfrentamento da pandemia, além do distanciamento físico, utilização de máscara e higienização de mãos e superfícies, informaram sobre a ventilação dos locais, pois gotículas contendo o vírus Sars Cov-2 podem ficar suspensas no ar, podendo contaminar pessoas saudáveis, portanto, os a ventilação do local deve se atentar em aumentar a circulação do ar externo, com abertura de portas e janelas e evitar o uso do ar condicionado e 
ventilador, mas, caso isso não seja possível é importante que o sistema de ar condicionado deve ter os dutos e filtros limpos, com limpeza semanal. Abrasel (2020c) recomenda que as mesas do tenham um distanciamento de 2 metros ou 1 metro entre as cadeiras. A Universidade Estadual de Campinas - UNICAMP (2020) também indica a utilização de home office para pessoas que fazem parte do grupo de risco, pois oferece menos risco de contaminação, indicam a retificação do layout no local de trabalho, com utilização de barreiras físicas de acrílico, indicação de distância no chão e parede e redução da capacidade total de pessoas.

Além disso, deve-se organizar o horário de refeição dos funcionários, para que haja segurança para todos. É indicado que os trabalhadores devem vacinar-se contra a gripo comum, que apesar de não servir para o Corona Vírus, reduz os casos de gripe (os sintomas podem ser confundidos com o da COVID 19). É importante que a higienização dos utensílios da cozinha seja realizada da maneira correta e os funcionários devem utilizar os Equipamentos de Segurança Individuais (EPIs), além de lavar a mãos corretamente antes de manusear objetos limpos e ao entregar para os clientes pratos e talheres, deve tomar cuidado para reduzir os riscos contato, os talheres podem ser protegidos dentro de embalagens plásticas e servidos apenas na hora do serviço, para evitar exposição dos mesmos (ABRASEL, 2020).

\section{CONSIDERAÇÕES FINAIS}

Identifica-se assim a evidente relevância da gestão da qualidade de higiene alimentar dos serviços de gastronomia de São Paulo, sobretudo com as expectativas de retomada dos negócios em 2021, com um excelente nível de sustentabilidade higiênico-sanitária, a recuperação e o desenvolvimento gradual das atividades socioeconômicas da alimentação ao longo do ano.

Porém, é sumamente necessário o desenvolvimento de uma forte parceria entre poder público municipal, empresários através de suas entidades oficiais, profissionais e acadêmicos do ramo. O episódio do Covid multiplicou em muito a necessidade e a possibilidade efetiva de prevenção e atualização permanente dos processos operacionais higiênicosanitários das cozinhas dos empreendimentos de gastronomia e hospitalidade. Dessa maneira, é chegada a hora e a vez, da implantação de programas de treinamento focados na criação e desenvolvimento de novos hábitos $e$ costumes de trabalho na produção de alimentos e a consequente adaptação das estruturas físicas e humanas existentes. Nesse momento, o papel dos líderes identifica-se como essencial para essa mudança e o avanço sustentável em Boas Práticas de qualidade em higiene. 
Para prestar um atendimento de qualidade, que atenda as expectativas dos clientes, é necessário ouvi-los. Além disso, é importante que os funcionários que atuam dentro dos estabelecimentos e com delivery devem receber treinamentos constantes a respeito do corona vírus, bem como como ocorre sua transmissão, afastamento em caso de sintomas, quais os sintomas que a COVID 19 causa, atender os clientes com cordialidade etc.

\section{REFERÊNCIAS BIBLIOGRÁFICAS}

ABRANCHES, M. V.; OLIVEIRA, C. T.; SANTOS, V. S. COVID -19: Estratégias para adaptação da produção, comercialização e gestão de estabelecimentos do setor de alimentos. São Carlos. ed. Scienza. 2020. Disponível em:

$<$ http://www.ntr.crp.ufv.br/wpcontent/uploads/2020/04/Ebook_COVID19_Estrat\%C3\%A9gias-paraadapta\%C3\%A7\%C3\%A3o-daprodu\%C3\%A7\%C3\%A3ocomercializa\%C3\%A7\%C3\%A3o-egest\%C3\%A3o-de-estabelecimentos-dosetor-de-alimentos1.pdf>. Acesso em: 10 jan 2021.

\section{ABRASEL - Associação Brasileira de} Bares e Restaurantes. Abrasel fecha acordo com iFood: prazo de pagamento para bares e restaurantes será reduzido. 2020a. Disponível em: <https://abrasel.com.br/noticias/noticias/ab rasel-fecha-acordo-com-ifood-prazo-depagamento-para-bares-e-restaurantessera-reduzido>. Acesso em 06 fev 2021.

ABRASEL - Associação Brasileira de Bares e Restaurantes. Cuidados de higiene no delivery para bares $e$ restaurantes na crise do novo coronavírus. 2020b. Disponível em: $<$ https://drive.google.com/file/d/1u3vSZDL AqDJeVTLROp5WM5WcTED1tpKV/view> . Acesso em: 06 fev 2021.

ABRASEL - Associação Brasileira de Bares e Restaurantes. Corona Vírus (COVID 19): Guia Prático Sobre como Lidar com o Problema em Bares e Restaurantes. 2020c. Disponível em: $<$ https://drive.google.com/file/d/1gnSFdzF 42fjpyKsoB6Ran7BLMiSgW4xo/view>. Acesso em: 18 fev 2021.

BRASIL. Decreto no 10.282, de 20 de março de 2020. Regulamenta a Lei $\mathrm{n}^{\circ}$ 13.979, de 6 de fevereiro de 2020, para definir os serviços públicos e as atividades essenciais. Brasília. 2020. Disponível em: <http://www.planalto.gov.br/ccivil_03/_ato 2019-2022/2020/decreto/D10282.htm>. Acesso em 15 fev 202.

BRASIL. Lei $\mathbf{n} \mathbf{0} \mathbf{1 3 . 9 7 9}$, de 6 de fevereiro de 2020. Dispõe sobre as medidas para enfrentamento da emergência de saúde 
pública de importância internacional decorrente do coronavírus responsável pelo surto de 2019. Brasília. 2020. Disponível em: <http://www.planalto.gov.br/ccivil_03/_ato 2019-2022/2020/lei/113979.htm>. Acesso em 15 fev 2021.

BRASIL. Ministério da Saúde. Resolução RDC n. 216, de 15 de setembro de 2004. Aprova o regulamento técnico de boas práticas para serviços de alimentação. Diário Oficial da União, Brasília, 2004. Disponível em: <https://bvsms.saude.gov.br/bvs/saudelegi s/anvisa/2004/res0216_15_09_2004.html> . Acesso em: 20 dez 2020.

CHEN, P. et al. Coronavirus disease (COVID-19): the need to maintain regular physical activity while taking precautions. Jour. Sport and Heal. Sci. vol 9, n. 2, p. 103-104. 2020. Disponível em: <https://www.ncbi.nlm.nih.gov/pmc/articles /PMC7031771/>. Acesso em: 06 fev 2021.

CHU, D. K. et al. Physical Distancing, Face Masks, and Eye Protection to Prevent Person-to-Person Transmission of SARSCoV-2 an COVID-19: a Systematic Review and Meta-analysis. The Lancet. Vol. 395, p. 1973-1987. 2020. Disponível em: $<$ https://www.thelancet.com/journals/lance t/article/PIIS0140-6736(20)31142-

9/fulltext\#articlelnformation>. Acesso em 19 fev 2021.
CRODAL, J. et al. COVID-19 in Brazil: advantages of a socialized unified health system and preparation to contain cases. Rev. Soc. Bras. Med. Trop. vol. 53. 2020. Disponível em: $<$ https://preprints.scielo.org/index.php/sciel o/preprint/view/7/14>. Acesso em: 13 jan 2021.

DONKOH, S. A. et al. Customer Satisfaction and Perceptions about Food Services on the University for Development Studies Campus, Ghana. Afr. J. Food Sci. v. 6, p. 216-223. 2012. Disponível em: $<$ https://academicjournals.org/article/articl e1380114368_Donkoh\%20et\%20al.pdf>. Acesso em: 05 jan 2021.

EI-SAID O.A.; FATHY E.A. Assessing university students' satisfaction with oncampus cafeteria services. Tour. Manag. Perspect. v. 16. 2015 Disponível em: $<$ https://www.researchgate.net/publication/ 325417620>. Acesso em: 02 fev 2021.

FAO (Organización de las Naciones Unidas para la Agricultura y la Alimentación); OMS (Organización Mundial de la Salud). COVID-19 e inocuidad de los alimentos: orientaciones para las empresas alimentarias. 2020. Disponível em: $<$ http://www.fao.org/3/ca8660es/CA8660E S.pdf>. Acesso em 8 fev 2021. 
\&

QUALIBEST

a.

Alimentação na Pandemia: A Visão dos

Operadores de Foodservice. 2020.

Disponível

em:

$<$ https://galunion.com.br/links-

galunion/materiais/pesquisa_alimentacao_ na_pandemia_galunion_anr_operadores3. pdf?utm_campaign=download_3_pesquis a_operadores\&utm_medium=email\&utm source $=R D+$ Station $>$. Acesso em: $18 \mathrm{fev}$ 2021.

\section{GALUNION \& QUALIBEST. Alimentação} na Pandemia: Como a Covid 18 impacta os consumidores e os negócios em alimentação. 2020. Disponível em: $<$ https://galunion.com.br/linksgalunion/materiais/pesquisa_alimentacao_ na_pandemia_galunion_onda3.pdf?utm_c ampaign=download_3_onda__pesquisa_alimentacao_na_pandemia_ga lunion_qualibest_-

_crise_covid19\&utm_medium=email\&utm _source=RD+Station $>$. Acesso em $18 \mathrm{fev}$ 2021.

KWUN, D. J. W. Effects of campus foodservice attributes on perceived value, satisfaction, and consumer attitude: A gender-difference approach. Int. J. Hosp. Manag. 2011. Disponível em: <https://www.academia.edu/1120295>. Acesso em: 15 jan 2021.

LU, J., et al. COVID-19 Outbreak Associated with Air Conditioning in
Restaurant, Guangzhou, China, 2020. Emerging Infectious Diseases. $2020 . \quad$ Disponível em: $<$ https://wwwnc.cdc.gov/eid/article/26/7/20 -0764_article\#tnF1>. Acesso em: $16 \mathrm{fev}$ 2021.

NADZIRAH, S. et al. University Food service: An Overview of Factors Influencing the Customers' Dining Choice. Int. Food Res. Journal. p. 1459-1468. 2013. Disponível em: $<$ http://www.ifrj.upm.edu.my/20\%20(03)\% 202013/63\%20IFRJ\%2020\%20(03)\%2020 13\%20Mohiddin\%20(222).pdf>. Acesso em: 10 jan 2021.

SÃO PAULO. Decreto no 12.342, de 27 de setembro de 1978. Aprova o Regulamento a que se refere o artigo 22 do Decreto-lei 211 , de 30 de março de 1970, que dispõe sobre normas de promoção, preservação e recuperação da saúde no campo de competência da Secretaria de Estado da Saúde. São Paulo Assembleia Legislativa do Estado de São Paulo. 1978a. Disponível em:

$<$ https://www.al.sp.gov.br/repositorio/legis| acao/decreto/1978/decreto-12342-

27.09.1978.html>. Acesso em: 13 jan 2021.

SÃO PAULO. Decreto no 12.486, de 20 de outubro de 1978. Aprova Normas Técnicas Especiais relativas a Alimentos e Bebidas. São Paulo: Assembleia Legislativa do Estado de São Paulo. 1978b. Disponível 
em:

$<$ https://www.al.sp.gov.br/repositorio/legis| acao/decreto/1978/decreto-12486-

20.10.1978.html>. Acesso em 13 jan 2021.

SÃO PAULO. Decreto no $\mathbf{6 4 . 8 8 1}$, de 22 de março de 2020. Decreta quarentena no Estado de São Paulo, no contexto da pandemia do COVID-19 (Novo Coronavírus), e dá providências complementares. São Paulo: Palácio dos Bandeirantes. 2020. Disponível em: $<$ http://dobuscadireta.imprensaoficial.com. br/default.aspx?DataPublicacao=2020032 $3 \&$ Caderno=DOE - I\&NumeroPagina $=1>$. Acesso em 15 fev 2021.

SÃO PAULO. Decreto no 64.994, de 28 de maio de 2020. Dispõe sobre a medida de quarentena de que trata 0 Decreto $\mathrm{n}^{-}$ 64.881, de 22 de março de 2020, institui o Plano São Paulo e dá providências complementares. São Paulo: Assembleia Legislativa do Estado de São Paulo. 2020. Disponível

em:

$<$ https://www.al.sp.gov.br/repositorio/legis| acao/decreto/2020/decreto-64994-

28.05.2020.html>. Acesso em 09 fev 2021.

SÃO PAULO. Decreto no 65.460, de 8 de janeiro de 2021. Altera os Anexos II e III do Decreto no 64.994, de 28 de maio de 2020 , que dispõe sobre a medida de quarentena de que trata o Decreto $n^{\circ} \mathbf{6 4 . 8 8 1}$, de 22 de março de 2020, e institui o Plano São Paulo. São Paulo: Assembleia Legislativa do Estado de São Paulo. 2021. Disponível em:

$<\mathrm{http}$ ://dobuscadireta.imprensaoficial.com. br/default.aspx? DataPublicacao $=2021010$ 9\&Caderno=DOE-I\&NumeroPagina $=1>$. Acesso em: 09 fev 2021.

SIEBENEICHLER et al. A satisfação de clientes de restaurantes: uma avaliação da satisfação e da importância dos atributos. Revista de Administração, Frederico Westphalen, v. 7, n. 11, p. 39-58. 2007. Disponível em: <http://revistas.fw.uri.br/index.php/revistad eadm/article/view/889>. Acesso em: 06 Fev 2021.

SPYRIDOU, A. Perceived Service Quality and Customer Revisiting Intention: The Case of "all you can eat" Asian Restaurants in Southern Taiwan. J. Tour. Herit. Serv. Mark. Vol. 3, n. 2, p. 30-38. 2017. Disponível em: <https://zenodo.org/record/1209129\#.YDB uQehKjIU>. Acesso em: 20 jan 2021.

STEFANINI, C. J.; ALVES, C. A.; MARQUES, R. B. Vamos almoçar? Um estudo da relação hospitalidade, qualidade em serviços e marketing de experiência na satisfação dos clientes de restaurantes. Rev. Bras. Pesq. Tur. vol. 12, n. 1, p. 5779, 2018. Disponível em: $<$ https://www.scielo.br/pdf/rbtur/v12n1/pt_ 1982-6125-rbtur-12-01-00057.pdf>. Acesso em 06 fev 2021. 
TAKALKAR, A. A.; KUMAVAT, A. P. Assessment of personal hygiene of canteen workers of government medical college and hospital, Solapur. Natl. J. Community Med. V. 2, p. 448- 451. 2011.

Disponível em:

$<$ http://www.njcmindia.org/uploads/2-

3_448-451.pdf >. Acesso em: 15 jan 2021.

UNICAMP - Universidade Estadual de Campinas. Orientações para o convívio seguro: Orientações Sanitárias para o Enfrentamento da Pandemia. Campinas. 2020. em:

<https://www.unicamp.br/unicamp/cartilhacovid-19/orientacoes-sanitarias-para-oenfrentamento-da-pandemia>. Acesso em 16 fev 2021.

WILDER-SMITH, A.; FREEDMAN, D. O. Isolation, quarantine, social distancing, and community containment: pivotal role for old-style public health measures in the novel coronavirus (2019-nCoV) outbreak. Jour. Trav. Med., v. 27, n. 2. Hamilton. 2020.

Disponível em:

$<$ https://ppgenfermagem.furg.br/images/C OVID_19/Artigos/15_Abril/Isolamento_e_ Medidas_de_Segurana_Pblica.pdf>. Acesso em: 01 fev 2021. 